\title{
Língua de sinais: visualizando a recepção da linguagem por meio da ressonância magnética funcional
}

Sign Language: visualizing the reception of language through functional magnetic resonance imaging

\author{
Michelle Nave Valadão \\ Universidade Federal de Viçosa \\ Myriam de Lima Isaac \\ Universidade de São Paulo \\ Sara Regina Escorsi Rosset ${ }^{1}$ \\ Universidade de São Paulo \\ Draulio Barros de Araujo \\ Universidade Federal do Rio Grande do Norte \\ Antonio Carlos dos Santos \\ Universidade de São Paulo
}

\section{Resumo}

As línguas de sinais são línguas naturais que compartilham as propriedades das línguas orais. Vários estudos demonstram que, durante o processamento das línguas de sinais, ocorre uma ativação cortical em áreas clássicas da linguagem. Este estudo buscou visualizar as áreas corticais ativadas em surdos sinalizadores, por meio de tarefa envolvendo a recepção da linguagem, através da língua de sinais. Foram realizados doze exames de imagem, por ressonância magnética funcional, por meio da utilização do aparelho de 1.5T (Siemens, Magneton Vision, Erlangen, Germany). Foi utilizada, ainda, uma sequência de imagem ecoplanar para a aquisição BOLD (Blood Oxygen Level Dependent) e, para corregistro, buscaram-se ima- gens de alta resolução de todo o encéfalo. Os mapas estatísticos foram obtidos com o mode- 
lo geral linear, utilizando-se o programa Brain Voyager ${ }^{\mathrm{TM}}$. Foram visualizadas ativações em áreas do córtex frontal, parietal e temporal nos participantes surdos sinalizadores. Tais descobertas sugerem que os campos receptivos da linguagem, nas línguas de sinais, são processados em regiões cerebrais homólogas aos engajados nas línguas orais.

\section{Palavras-chave}

língua brasileira de sinais; língua de sinais; linguagem; imagem por ressonância magnética funcional.

\section{Abstract}

Sign languages are natural languages that have the same properties of oral languages. Several studies have demonstrated activation of the classical language areas during sign languages processing. This study aimed to identify the cortical areas activated in deaf subjects who communicate via Brazilian Sign Language in a receptive language task. Twelve functional magnetic resonance imaging (fMRI) were performed using a $1.5 \mathrm{~T}$ equipment (Siemens, Magneton Vision, Erlangen, Germany). Echo Planar Imaging (EPI) sequence was used to obtain BOLD (Blood Oxygen Level Dependent), associated with a whole brain high resolution imaging for co-registering. The statistical maps were obtained using General Linear Model with Brain Vyyager $^{\mathrm{TM}}$ software. For deafs, the sign perception task produced activations in the frontal, temporal and parietal cortex regions. These data suggest that sign languages are processed bilaterally in classical language regions in the same way as spoken languages are.

\section{Keywords}

brazilian sign language (BSL); sign language; language; functional magnetic resonance imaging. 


\section{Introdução}

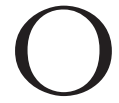

reconhecimento das línguas de sinais como línguas naturais forneceu uma oportunidade de pesquisa para a compreensão e para o entendimento da base neural relacionada à organização da linguagem no cérebro humano. Uma vez que essas línguas possuem níveis de organização linguística idênticos aos das línguas orais auditivas (como sistemas fonológico, morfológico, sintático, semântico, etc.), ainda que os falantes usem as mãos como principais articuladores e a visão como canal de percepção da linguagem, despertou-se o interesse em investigar os possíveis substratos neurais que se encontram associados ao processamento da linguagem por meio das línguas de sinais.

Assim, se a neuroanatomia funcional das línguas orais fosse determinada exclusivamente pela produção e pela percepção dos sons, as línguas de sinais poderiam apresentar padrões distintos de organização cerebral. Com o advento dos exames de neuroimagem, especialmente das imagens por ressonância magnética funcional (IRMf), tornou-se possível compreender melhor os mecanismos envolvidos nos diferentes processos mentais relacionados às línguas de sinais.

Em relação a elas, os exames em neuroimagem possibilitam a identificação das regiões relacionadas ao processamento da linguagem no córtex cerebral, permitindo o estabelecimento de semelhanças e diferenças quando na comparação desse processamento aos processos envolvidos nas línguas orais.

Dessa forma, este artigo descreve os achados de um pioneiro estudo brasileiro que investigou a representação cortical no processamento da língua brasileira de sinais. De modo que seu objetivo central foi utilizar a IRMf a fim de se identificarem áreas de ativação cortical, durante o desenvolvimento de atividade de recepção da linguagem, por meio da língua brasileira de sinais (Libras). 


\section{Imagem por ressonância magnética funcional como ferramen- ta para a identificação dos processos mentais envolvidos nas lín- guas de sinais}

A linguagem pode ser definida como a capacidade para compreender e usar, em modalidades diversas de comunicação e pensamento, um sistema complexo e dinâmico de símbolos convencionados (AMERICAN SPEECH AND HEARING ASSOCIATION, 1982). Ela se manifesta em todas as atividades sociais e culturais, constituindo a base da transmissão cultural, dos sistemas de valores e das tradições que caracterizam a sociedade humana. Está ligada à experiência do homem de tal forma que se torna impossível imaginar a vida sem ela, uma vez que se constitui como instrumento essencial para aceder aos pensamentos e conhecimentos. Não obstante, pressupõe o uso de um sistema codificado, o qual, quando está enraizado e é frequentemente utilizado por dada comunidade, é percebido como uma língua.

O conceito de linguagem reflete um sentido mais amplo que o conceito de língua: a linguagem é um sistema de comunicação natural ou artificial, humano ou não humano; enquanto a língua, por sua vez, é vista como um sistema abstrato de regras gramaticais, as quais podem ser concebidas como convenções sociais, construídas pelas comunidades humanas, e/ou como um sistema estruturado de signos que exprimem determinadas ideias (FERNANDES, 1998).

Língua não se confunde com linguagem: é somente uma parte determinada, essencial dela, indubitavelmente. É, ao mesmo tempo, um produto social da faculdade de linguagem e um conjunto de convenções necessárias, adotadas pelo corpo social para permitir o exercício dessa faculdade nos indivíduos. (SAUSSURE, 1995)

Segundo a Linguística, ciência que se ocupa de estudar as línguas naturais, a linguagem é regida por princípios que fazem parte do conhecimento humano e que determinam a produção das palavras, a construção das sentenças 
e dos textos. Desse modo, tem-se que os princípios expressam as generalizações e as regularidades da linguagem segundo os diferentes níveis em que ela é utilizada (QUADROS; KARNOPP, 2004).

De acordo com as autoras citadas, outro pressuposto dos estudos linguísticos é a universalidade dos princípios, ou seja, independentemente do estudo de uma língua específica, é possível determinar os princípios universais que regem todas as línguas, uma vez que as investigações referentes aos aspectos específicos de cada língua revelam as características da linguagem humana. Assim, apesar das diferenças existentes entre as línguas, as estruturas apresentam aspectos comuns que explicam a natureza da linguagem humana.

No que diz respeito aos estudos linguísticos referentes às línguas de sinais, as primeiras pesquisas surgiram em torno da década de 1960, quando Stokoe (1960) apud Moura, Lodi e Harrison (2005, p. 353), linguista americano, descreveu a estrutura da língua americana de sinais, a partir de seus elementos constituintes. Através disso, comprovou-se que ela atendia a todos os critérios linguísticos de uma língua genuína: em termos de léxico, de sintaxe e de capacidade para gerar uma quantidade infinita de sentenças. Stokoe observou ainda que os sinais não eram imagens, mas símbolos abstratos complexos, que continham uma estrutura interior.

Segundo Souza (1998), Stokoe empenhou-se em evidenciar a isomorfia entre sinal e fala. O linguista nomeou de "quirema" o segmento mínimo sinalizado, o qual seria correspondente ao fonema da fala. Segundo Stokoe, cada morfema, unidade mínima de significação, seria composto por três quiremas: ponto de articulação, configuração das mãos e movimento.

No Brasil, a língua de linais é denominada língua brasileira de sinais (Libras) (BRASIL, 2002). Alguns estudos linguísticos brasileiros procuraram estabelecer as unidades formadoras dos sinais e o conjunto de traços distintivos de unidades mínimas, detalhando sua representação fonológica. Tais estudos demonstraram que a Libras, assim como as outras línguas de sinais, possuem as mãos como articuladores primários, de modo que elas se movimentam no espaço, em frente ao corpo, e articulam sinais em determinadas locações nesse mesmo espaço. Movimentos do corpo e da face também desempenham funções 
e seus principais parâmetros fonológicos são locação, movimento e configuração de mão (FERREIRA-BRITO, 1990; QUADROS; KARNOPP, 2004).

Trabalhos, que descreveram detalhadamente como articular o sinal, especificaram os seguintes elementos: a articulação da(s) mão(s) e dos braços, a orientação das palmas, o local dessa articulação em relação ao corpo, o movimento no espaço da sinalização e a expressão facial associada (CAPOVILLA; RAPHAEL; LUZ, 2001; CAPOVILLA et al., 2002).

Com base nesses estudos, é consenso afirmar que a língua de sinais é considerada uma língua natural para os surdos. De acordo com Capovilla, Capovilla e Macedo (2006), a língua de sinais, como língua primária, desenvolve-se de modo natural desde que as capacidades visuais e manuais sejam preservadas.

Assim, em virtude de possuir características visuoespaciais, não há barreira de natureza perceptual para a sua aquisição. Entretanto, é necessário que o indivíduo surdo, desde a mais tenra idade, esteja imerso em uma comunidade surda sinalizadora, a fim de que, naturalmente, a língua de sinais seja adquirida (CAPOVILLA et al., 2004).

O reconhecimento do valor linguístico das línguas de sinais forneceu uma importante oportunidade de pesquisa para o entendimento das áreas corticais envolvidas no processamento das mesmas no cérebro humano. O primeiro avanço, nessa área, ocorreu através da publicação de um estudo que descrevia casos clínicos de alterações da linguagem em indivíduos surdos, nativos na língua de sinais, que sofreram lesões cerebrais (POIZNER; KLIMA; BELLUGI, 1987 apud CAMPBELL; MACSWEENEY; WATERS, 2008).

Os autores citados relataram que os indivíduos surdos que sofreram danos em regiões frontais do hemisfério cerebral esquerdo apresentavam dificuldades em se expressar na língua de sinais. Essas dificuldades eram semelhantes às alterações de expressão da fala (afasia de Broca) encontradas em ouvintes que sofriam lesões em regiões cerebrais semelhantes. Da mesma forma, lesões em regiões temporais do hemisfério cerebral esquerdo ocasionavam alterações na compreensão da língua de sinais, assim como dificuldades em compreender a fala e afasia de Wernicke em ouvintes.

Por outro lado, os estudos envolvendo indivíduos surdos afásicos foram 
fundamentais para o estabelecimento de modelos que pudessem demonstrar os mecanismos cerebrais envolvidos no processamento cerebral da língua de sinais. Entretanto, somente com o desenvolvimento e o acesso às técnicas de neuroimagem é que se tornou possível detalhar e caracterizar os sistemas neurais que são ativados durante a percepção e a cognição do cérebro humano intacto.

É possível observar imagens do cérebro em funcionamento por meio de técnicas de neuroimagem funcional. A principal delas é a imagem funcional por ressonância magnética (IRMf), a qual vem sendo uma das principais fontes de geração de imagens do cérebro em funcionamento na última década. Seu caráter não invasivo e sua boa resolução temporal têm contribuído para seu avanço e implementação (LOGOTHETIS, 2002; OGAWA et al., 1990a; OGAWA et al., 1990b; STURZBECHER, 2006).

Segundo Amaro Junior e Barker (2006), a IRMf constitui-se como um método muito poderoso para investigar o cérebro em respostas a testes cognitivos. O aumento da utilização dessa técnica se deve à sua flexibilidade, disponibilidade, alta resolução espacial, a uma resolução temporal relativamente alta e à falta de radiações ionizantes ou da necessidade de agentes de contraste externos.

A IRMf utiliza, basicamente, o fenômeno de ressonância magnética e o nível de oxigenação da hemoglobina para evidenciar a atividade neuronal de uma maneira indireta, através da resposta hemodinâmica induzida pela ação - como falar ou mexer a mão (HUETTEL; SONG; MCARTHY, 2004).

A atividade neuronal promove um discreto aumento local do metabolismo cerebral e do consumo de oxigênio. Esse fenômeno é seguido de um aumento da perfusão cerebral local; a qual é responsável por uma elevação do transporte de oxi-hemoglobina, que ultrapassa o consumo de oxigênio, e resulta em uma hiperoxigenação sanguínea local, no capilar e em volta desse, podendo ser detecta pela IRMf.

Essa técnica faz uso do chamado efeito BOLD (Blood Oxygen Level Dependent) (ROSSET, 2008). O efeito BOLD deve-se às mudanças locais na proporção de oxi e deoxi-hemoglobina, o que ocorre em virtude do aumento da variação do fluxo sanguíneo e da demanda de oxigênio, por parte do tecido, ante a ativação neuronal (OGAWA et al., 1990a; OGAWA et al., 1990b). 
Assim, por meio dessa importante técnica de neuroimgem funcional, foi possível ampliar o conhecimento a respeito da localização cerebral das áreas de linguagem. Em relação aos surdos profundos, a IRMf demonstra ser um importante recurso para a compreensão da linguagem humana: através da exploração dos sistemas neurais que sustentam o processamento da linguagem em suas diversas formas, uma vez que, independentemente das suas diferentes modalidades (oral-auditiva ou visuo-manual), as línguas são regidas por universais linguísticos.

Um estudo pioneiro em IMRf e em surdez foi desenvolvido por Neville et al. (1998). Nesse estudo, os cientistas buscaram caracterizar a organização cerebral de indivíduos surdos, durante o processamento da língua de sinais, em comparação com indivíduos ouvintes. Os resultados mostraram que tanto os surdos quanto os ouvintes, quando realizavam tarefas de linguagem em suas línguas nativas, apresentaram ativações em áreas clássicas da linguagem no hemisfério cerebral esquerdo.

Nessa mesma linha de pesquisa, MacSweeney et al. (2002a) ressaltaram que alguns padrões de localização da linguagem são independentes da modalidade da língua, enquanto outros são dependentes. Em relação aos padrões independentes, os pesquisadores encontraram, em regiões corticais, ativações similares tanto em surdos sinalizadores quanto em ouvintes, durante a realização de tarefas em suas línguas nativas.

Tais ativações estiveram presentes em regiões pré-frontais inferiores (incluindo a área de Broca) e em regiões temporais superiores (incluindo a área de Wernicke). Em relação aos padrões que dependem da modalidade específica da língua, a língua inglesa, para os ouvintes, gerou maior ativação no córtex auditivo primário e secundário, enquanto a língua de sinais gerou maior ativação em regiões do córtex visual.

Dessa forma, a IRMf mostra-se uma importante ferramenta a ser utilizada para o entendimento da organização neural no processamento das línguas de sinais, de modo que a visualização das áreas corticais, ativadas durante tarefa de recepção da linguagem em língua de sinais, é o foco deste trabalho. 


\section{Metodologia}

Foram realizados exames de IRMf em um grupo composto por doze participantes surdos, dos quais oito pertencem ao gênero feminino e quatro ao gênero masculino. Os participantes apresentam uma média de 25,41 anos de idade e 11,25 anos de escolaridade. Todos apresentavam perda auditiva sensorioneural (SN), de grau profundo bilateralmente (limiares audiométricos tonais acima de noventa e um dBNA para as frequências de 500, 1000 e $2000 \mathrm{~Hz}$ - segundo Davis, 1970), e se comunicavam exclusivamente através da Libras. Os sujeitos pesquisados assinaram um termo de consentimento livre e esclarecido, tendo a liberdade de aceitar ou não participar dessa pesquisa e podendo, a qualquer momento, retirar-se do estudo, sem que isso acarretasse algum prejuízo ou dano a eles. O estudo foi desenvolvido pelo Departamento de Neurociências e Ciências do Comportamento da Faculdade de Medicina de Ribeirão Preto da Universidade de São Paulo e aprovado pelo Comitê de Ética em Pesquisa, do Hospital das Clínicas da Faculdade de Medicina de Ribeirão Preto.

O equipamento utilizado foi um aparelho de ressonância magnética Siemens, modelo Magneton Vision, de 1.5 Tesla, com bobina de cabeça de quadratura e polarização circular comercialmente disponível.

O estímulo foi exposto aos participantes da pesquisa através de gravação visual digital e consistiu na apresentação de um filme no qual um ator, um indivíduo surdo do gênero masculino, narrou uma história (mais especificamente uma fábula) em Libras. Os participantes surdos receberam uma orientação para que ficassem atentos e buscassem compreender o conteúdo da história. O vídeo foi projetado, por meio de um projetor digital, em uma tela translúcida, a qual foi verticalmente colocada em posição próxima aos pés dos participantes. Os mesmos permaneceram deitados dorsalmente no scanner e assistiram ao filme olhando para a tela, através de um espelho acoplado à cabeça.

O paradigma de bloco também foi utilizado. Ele consistiu em 11 blocos, divididos em cinco estados ativos intercalados por seis estados de repouso, com média de 27,5 segundos cada, de modo que o tempo total do exame resultou em aproximadamente cinco minutos. Os estímulos, que diferenciavam os pe- 
ríodos de atividade e de repouso, foram apresentados por meio de gravação digital visual. Para os períodos de atividade, os participantes foram instruídos a ficarem atentos ao conteúdo da história. Para os intervalos de repouso, cujo intuito foi o de interromper a atividade cerebral gerada pela tarefa solicitada (período de atividade), os participantes foram instruídos a não pensarem em nada ou pensarem em uma parede branca.

Para a série de imagens funcionais, foi utilizada uma sequência de imagem ecoplanar para aquisição BOLD. Como a imagem possui uma resolução de menor qualidade, fez-se necessária a utilização de uma imagem de alta resolução espacial. Assim, foram empregadas imagens sagitais ponderadas em T1, cobrindo ambos os hemisférios cerebrais e utilizando uma sequência do tipo gradiente eco 3D de alta resolução. Após a aquisição das imagens, elas foram processadas por meio de um programa comercial (BrainVoyager QX, Brain Innovation, Maastricht, The Netherlands).

Quanto aos resultados da IRMf, a análise estatística foi baseada no Modelo Geral Linear. Nesse método, a evolução temporal da intensidade de cada pixel foi comparada a uma função de referência modelada do sinal, verificandose a similaridade entre ambas. Assim, foram observados os pixels que apresentaram comportamento mais próximo do esperado. As visualizações dos mapas estatísticos foram feitas pela sua superposição em imagens de boa resolução anatômica, provenientes de sequência T1 volumétrica.

Os mapas de ativação obtidos nos estudos funcionais para cada indivíduo foram transformados em um espaço comum de coordenadas: o espaço Talairach (TALAIRACH; TOURNOUX, 1988). Esse sistema de coordenadas facilita a comparação direta entre os resultados de localização funcional e, por ser uma ferramenta de correção das variações anatômicas cerebrais entre os sujeitos estudados (JUSTINA, 2005), também supre a necessidade de comparação interindividual.

A significância estatística foi corrigida, para múltiplas comparações, por meio da False Discovery Rate, a qual tem como objetivo principal colocar a taxa de falsos positivos para $\alpha$ de 0,05 . Visando-se a garantir a consistência dos resultados, realizou-se um procedimento de agrupamento ou "clusterização" dos 
voxeis com diferenças estatísticas significativas: nesse trabalho, os clusters deveriam possuir mais de 50 voxeis.

Uma vez que o maior interesse dos estudos em neuroimagem é a apresentação de resultados que representem estudos populacionais, após a obtenção dos dados de cada indivíduo, a próxima etapa se referiu à geração do estudo de grupo, ou seja, à análise das áreas comumente ativas para todos os participantes do grupo; as quais também foram colocadas no espaço Talairach.

Para essa análise estatística, utilizou-se, ainda, o artifício Random effect, uma análise intersujeito que assume o efeito do experimento como variável entre os indivíduos. As séries temporais de cada participante foram traduzidas em mapas estatísticos, os quais passaram por uma segunda etapa de análise, gerando o mapa estatístico combinado (HUETTEL; SONG; McCARTHY, 2004).

A análise de grupo foi realizada através do Modelo Geral Linear, por meio do programa Brain Voyager. Nessa análise, foram selecionadas, considerando-se todos os participantes do grupo, as regiões corticais predominantemente ativadas, e as imagens foram escolhidas de modo a ilustrar as principais áreas corticais, áreas de Brodmann (BA), ativadas no estudo de grupo. Na descrição dos resultados, as áreas de comum ativação, encontradas em todo o grupo, foram representadas, para visualização, através de imagens da cabeça de apenas um dos participantes.

\section{Resultados}

Em relação às áreas corticais classicamente envolvidas nos processos da linguagem, foram encontradas ativações em área parietotemporal associativa (BA 39, 40, 7, 21, 22, 37), cuja função está relacionada à integração sensorial entre a visão e a mão, a visão e a memória, a visão e o tato, além das relações espaciais. As ativações abrangeram a área de Wernicke (BA 39 e 40), região classicamente envolvida na compreensão da linguagem.

Além disso, foram encontradas ativações em área visual associativa dos lobos parietal, occipital e temporal (BA 18, 19), os quais desempenham funções ligadas à análise do movimento, ao controle e à fixação visual e à memória vi- 
sual. Na tabela 1, são demonstradas as principais áreas corticais (giros e áreas de Brodmann) e seus respectivos números de voxeis ativos.

Tabela1: Áreas corticais e número de voxeis ativos

\begin{tabular}{lrrrr}
\hline \multicolumn{1}{c}{ Giros } & \multicolumn{2}{c}{ Areas de Brodmann } & \multicolumn{2}{c}{ Voxeis } \\
& \multicolumn{1}{c}{ HCD } & \multicolumn{1}{c}{ HCE } & HCE & HCD \\
\hline Giro temporal superior & 42413839222113 & 42413839222113 & 1542 & 1856 \\
Giro temporal médio & 222137391938 & 2221373919 & 2185 & 1809 \\
Ínsula & 41132240 & 13 & 339 & 278 \\
Giro temporal inferior & 203719 & 20371921 & 441 & 232 \\
Giro fusiforme & 37361920 & 3736191820 & 584 & 786 \\
Giro occipital médio & 193718 & 193718 & 517 & 151 \\
Lóbulo parietal inferior & 440 & 402 & 359 & 745 \\
Giro frontal inferior & 459461047 & 45 & 34 & 738 \\
& & & & \\
\hline
\end{tabular}

HCE: Hemisfério Cerebral Esquerdo

HCD: Hemisfério Cerebral Direito

Nas figuras a seguir (figuras 1, 2, 3, 4 e 5), são apresentadas algumas imagens nas quais é possível visualizar as principais regiões corticais ativadas, pelos participantes, durante a realização da tarefa:
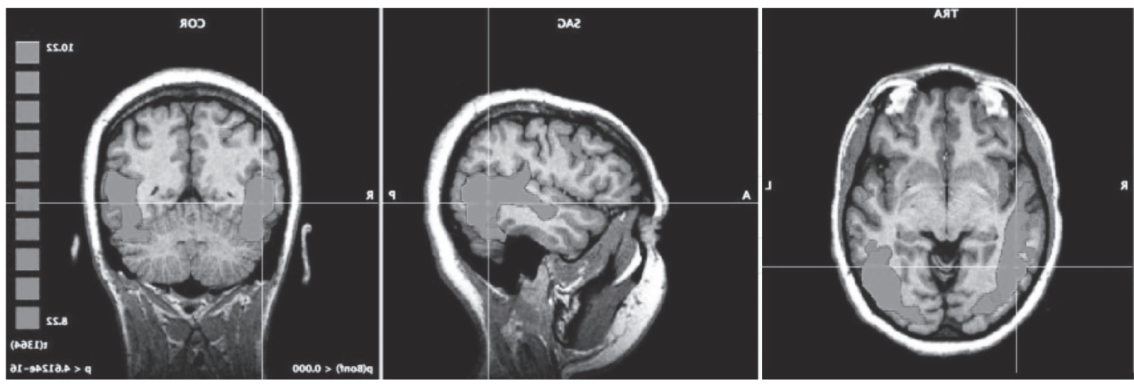

Figura 1: Imagem mostrando ativação em BA18, BA 19, BA 39 e BA 22.
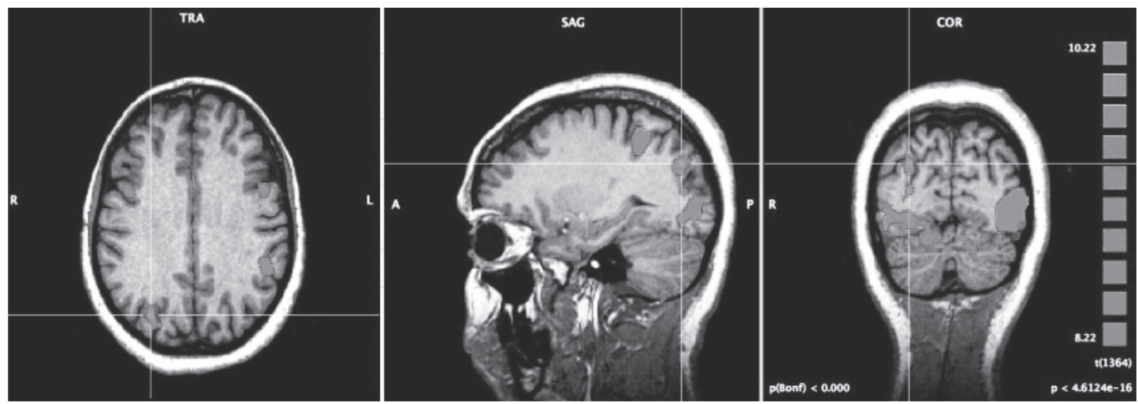

Figura 2: Imagem mostrando ativação em BA 7. 

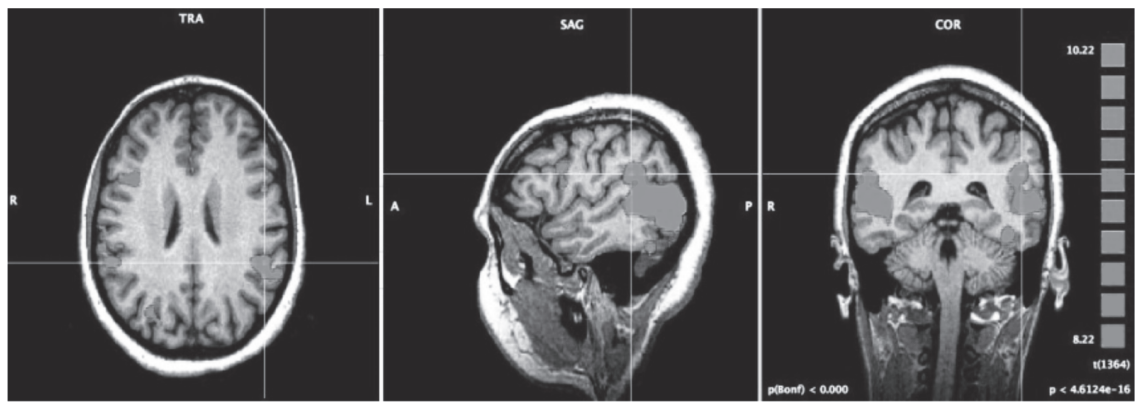

Figura 3: Imagem mostrando ativação em BA 40.
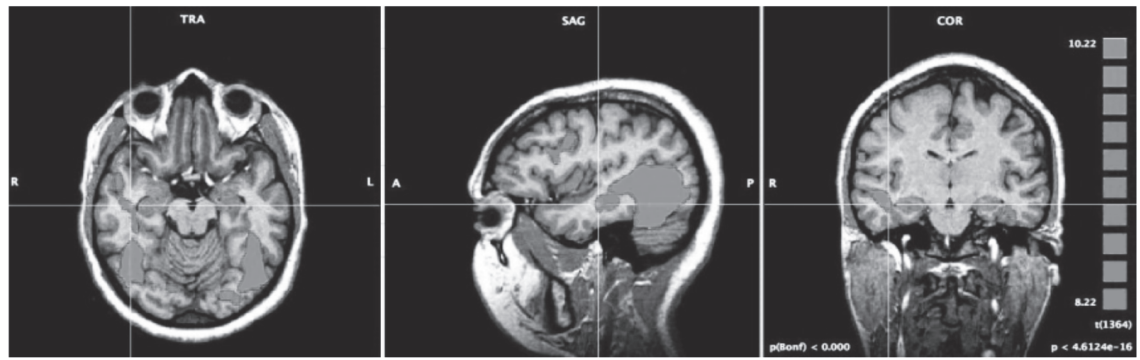

Figura 4: Imagem mostrando ativação em BA 21.
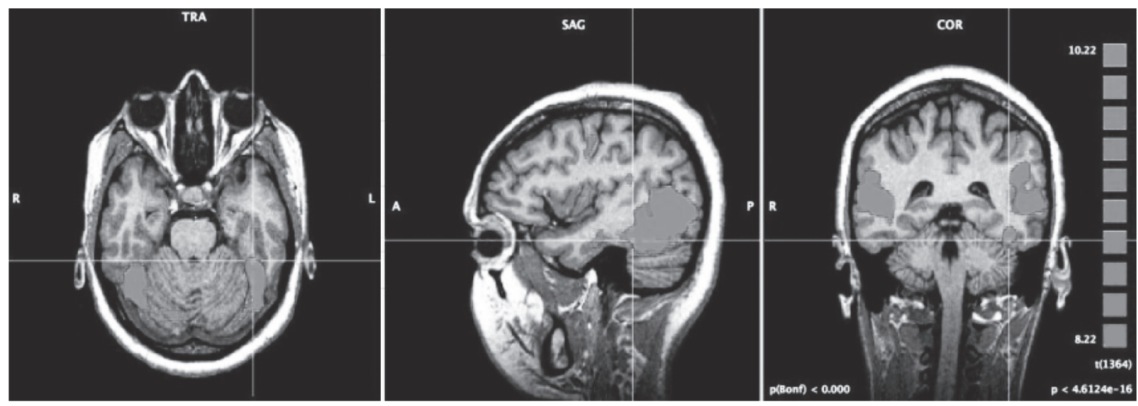

Figura 5: Imagem mostrando ativação em BA 37.

\section{Discussão}

As ativações encontradas em nosso trabalho refletem o envolvimento da compreensão da língua de sinais em relação aos surdos sinalizadores. Esse aspecto ativou regiões do giro fusiforme, da ínsula, dos giros temporais superior, médio e inferior, incluindo a área de Wernicke e estendendo-se ao giro frontal inferior (BA 
18, 19, 21, 22, 37, 39, 40); além de eliciar ativação em regiões parietais (BA 7).

As regiões temporais estão tradicionalmente relacionadas às tarefas que envolvem a compreensão da língua oral por ouvintes - como a escuta de uma história. Assim, pode-se observar que, nos surdos sinalizadores, ocorreram ativações de maneira homóloga para a compreensão da língua de sinais. Esse dado permite inferir que existe uma utilização da área de Wernicke, que é referente à tarefa de compreensão da linguagem em Libras.

As ativações no giro temporal médio posterior estão relacionadas ao processamento de movimento, enquanto as ativações na região do lobo parietal, especialmente BA 7, sugerem determinada relação de representação corporal no que diz respeito ao processo de recepção da língua de sinais.

De acordo com Hickok, Bellugi e Klima (2001), apesar de as línguas de sinais envolverem processamentos visuais, sua organização neural compartilha mais elementos comuns com as línguas orais do que com os processamentos visuoespaciais, ainda que as línguas difiram em termos de modalidade. Embora, nos estágios iniciais de processamento, os sistemas neurais, responsáveis por cada uma delas, envolvam vias diferentes, uma vez ultrapassados esses estágios iniciais, elas compartilham o mesmo substrato neural.

Em estudos pioneiros sobre neuroimagem, Söderfeldt, Rönnberg e Risberg (1994) e Söderfeldt et al. (1997) compararam a ativação cortical para a tarefa de compreensão em surdos sinalizadores e em ouvintes, contrastando a língua de sinais sueca e a língua oral. O primeiro estudo não encontrou nenhuma diferença significativa entre as duas línguas. Entretanto, o último estudo demarcou divergências entre as modalidades de línguas, as quais ocorreram, basicamente, em regiões que são especializadas em diferentes modalidades de entrada e não em regiões típicas de processamento de linguagem. O lóbulo temporal superior, região do córtex auditivo, foi mais ativado pela língua oral, enquanto o córtex temporal inferior e o córtex occipital, relacionados às regiões visuais, foram mais ativados pela língua de sinais.

Podemos dizer que as tarefas que demandam compreensão também são ricas em informações narrativas, visto que, ao assistirem ao filme, os participantes permaneceram atentos ao conteúdo narrado; no qual se encontravam im- 
plícitas informações pragmáticas da linguagem que, no caso das línguas de sinais, foram percebidas por meio de expressões faciais e movimentos corporais.

Para as línguas de sinais, essas informações são extremamente necessárias à compreensão das informações transmitidas, pois um mesmo sinal pode representar diferentes signos linguísticos, diferindo apenas na posição espacial em que ele é apresentado ou na expressão facial e/ou corporal que o acompanha.

Com base nesses dados, Newman et al. (2010) argumentaram que a modalidade visuomanual da língua de sinais possui recursos linguísticos que não estão disponíveis para as línguas orais, tais como posições das mãos e movimentos corporais no espaço, além de expressões faciais, etc.

O padrão de ativação para o processamento de informação narrativa encontrado em nosso estudo foi similar aos encontrados nos estudos de IRMf sobre línguas orais avaliadas por meio de protocolos que envolviam a fala (MEYER et al., 2002; MITCHELL et al., 2003; NARUMOTO et al., 2001), o que sugeriu que tanto as línguas de sinais quanto as línguas orais possuem um circuito neuronal comum para o processamento de informações narrativas.

No que se refere especificamente à língua de sinais e ao processamento de informações narrativas (prosódia, fluência, expressão facial) nessa língua, Newman et al. encontraram ativações corticais em regiões perisilvianas e em gânglios da base, em regiões frontais médias e em regiões temporais mediais.

Um importante achado científico refere-se à ativação do giro fusiforme, denominado de área fusiforme da face. Trabalhos que avaliaram a expressão facial acompanhando a língua de sinais encontraram ativação nessa região como respostas às expressões faciais emocionais e linguísticas (MCCULLOUGH; EMMOREY; SERENO, 2005; NEWMAN et al).

A presença de ativação no giro temporal superior corrobora trabalhos precedentes que discutiram o fato de que as informações narrativas, das línguas de sinais, apresentavam predomínio de expressões faciais e de movimentos corporais associados ao caráter espacial da língua. Provavelmente, todas essas funções são usadas na interpretação do discurso narrativo, e supõe-se que funções cognitivas múltiplas ocorram em regiões do sulco temporal superior, as quais dependem da tarefa atribuída e das interações com outras regiões cerebrais 
(HEIN; KNIGHT, 2008). A ativação na área fusiforme da face também demonstra que, na língua de sinais, as expressões faciais são essenciais para a decodificação linguística dos sinais.

De acordo com as suas particularidades e na busca por se definirem os diversos fatores envolvidos no processamento da língua de sinais, diversas hipóteses têm sido testadas segundo diferentes variáveis. Como já mencionamos, uma das características da língua de sinais é a existência da relação entre os sinais e a posição em que os mesmos são executados em frente ao corpo. Algumas sentenças em sinais utilizam esse espaço para estabelecer relações entre elas, de forma que alguns estudos as denominam como sentenças topográficas.

Usando a IRMf, MacSweeney et al. (2002b) estudaram o processamento de sentenças topográficas em sinais em áreas corticais de surdos e de ouvintes bilíngues em BSL. Quando os participantes executaram uma tarefa que demandava o julgamento de anomalias na sentença, a região occipitotemporal foi ativada, em maior medida, para as sentenças topográficas do que para o processamento de sentenças não topográficas.

A ativação foi observada, nos lobos parietais superior e inferior esquerdo, durante o processamento de sentenças topográficas em língua de sinais. Dessa forma, os achados de MacSweeney et al. sugeriram que o lóbulo parietal esquerdo estaria envolvido, especificamente, no processamento da configuração e das posições precisas das mãos no espaço para representação de objetos e de ações. Apesar da diferença de protocolos aplicados, observamos, em nosso estudo, ativações no lobo parietal inferior, durante a realização da tarefa de recepção da linguagem em língua de sinais, do grupo de surdos sinalizadores.

\section{Conclusões}

A IRMf, pela técnica $B O L D$ mostrou ser uma importante ferramenta para a visualização dos campos receptivos da linguagem por meio da Libras.

Nossos achados demonstraram concordância com a literatura no que se refere à existência de um substrato cortical comum de ativação entre a língua de sinais e as línguas orais - para a função de compreensão da linguagem, com o en- 
volvimento de regiões do córtex temporal superior, incluindo-se a área de Wernicke.

\section{Referências}

AMARO JUNIOR, Edson; BARKER, Gareth J. Study design in fMRI: Basic principles. Brain and Cognition, v.60, n.3, p. 220-232, 2006.

AMERICAN SPEECH AND HEARING ASSOCIATION (1982). Disponível em: http:// www.asha.org/docs/html/RP1982-00125.html Acesso em: 21 abr. 2011.

BRASIL. Lei n. 10406, de 10 de janeiro de 2002. Institui o Código Civil brasileiro. Disponível em: http://trtapl3.trt12.gov.br/cmdg/img_legis/2002/ 01100610406. html Acesso em: 04 mar. 2006.

CAMPBELL, Ruth; MACSWEENEY, Mairéad; WATERS, Dafydd. Sign language and the Brain: a review. Journal of Deaf Studies and Deaf Education, v.13, n.1, p. 3-20, 2008.

CAPOVILLA, Fernando Cesar; RAPHAEL, Walkíria Duarte; LUZ, Renato Dente. Capítulos introdutórios. IN: CAPOVILLA, Fernando Cesar; RAPHAEL, Walkíria Duarte. Dicionário Enciclopédico Ilustrado Trilingue da Lingua de Sinais Brasileira. Sinais de M a Z. , v. II. 2 ed. São Paulo: Editora da Universidade de São Paulo - Imprensa Oficial do Estado, 2001.

CAPOVILLA, Fernando Cesar et al. Enciclopédia digital da Língua de Sinais Brasileira e sistemas de indexação e busca de sinais baseadas em menus quirêmicos. Espaço: Informativo Técnico-Científico do Instituto Nacional de Educação e Integração de Surdos, v. 17, 2002.

CAPOVILLA, Fernando Cesar et al. Avaliando compreensão de sinais da Libras em escolares surdos do ensino fundamental. Interação em Psicologia, v.8, n.2, p. 159-169, 2004.

CAPOVILlA, Fernando Cesar; CAPOVILlA, Alessandra Gotuzo Seabra; MACEDO, Elizeu Coutinho de. Recursos de reabilitação de distúrbios da comunicação e linguagem para melhor qualidade de vida em quadros sensoriais, motores e cognitivos. O Mundo da Saúde, v. 30, n. 1, p. 26-36, 2006.

DAVIS, Hallowell. Abnormal hearing and deafness. In: DAVIS, Hallowell; SIL- 
VERMAN, Sol Richard. Hearing and deafness. $3^{\text {Th }}$ ed. New York: Rinehart and Winston, 1970.

DE ARAÚJO, Draulio Barros. Sobre neuroimagens funcionais por magnetoencefalografia e ressonância magnética: novos métodos e aplicações. Ribeirão Preto: USP, 2002. Tese (Doutorado) - Faculdade de Filosofia Ciências e Letras da Universidade de São Paulo, Ribeirão Preto, 2002.

FERNANDES, Eulália. Teorias da aquisição da linguagem. IN: GOLDFELD, Márcia. Fundamentos em fonoaudiologia. Rio de Janeiro: Guanabara Koogan S.A, 1998. FERREIRA-BRITO, Lucinda. Uma abordagem fonológica dos sinais da LSCB. Espaço: Informativo Técnico Científico do INES, v.1, n.1, p. 20-43, 1990.

HEIN, Grit; KNIGHT, Robert T. Superior temporal sulcus - it's my area: or is it? Journal of Cognitive Neuroscience, v.20, n.2, p. 2125-2136, 2008.

HICKOK, Gregory Scott; BELLUGI, Ursula; KLIMA, Edward S. Sign language in the brain. Scientific American, v.284, n.6, p. 58-65, 2001.

HUETTEL, Scott A.; SONG, Allen W.; McCARTHY, Gregory. Functional Magnetic Ressonance Imaging, 1ํㅡㄹ $\mathrm{ed}$. Sunderland: Sinauer Associates, 2004.

JUSTINA, Helen Mathei Della. Variabilidade da atividade cerebral em resposta a estímulos vestibular e oculomotor avaliada por fMRI. Ribeirão Preto: USP, 2005. Dissertação (Mestrado) - Faculdade de Filosofia Ciências e Letras da Universidade de São Paulo, Ribeirão Preto, 2005.

LOGOTHETIS, Nikos K. The neural basis of the blood-oxigen-level-dependent functional magnetic resonance imaging signal. Philosophical Transactions of the Royal Society Biology Science, v.357, n.1424, p.1003-1037, 2002.

MACSWEENEY, Mairéad et al. Neural systems underlying British Sign Language and audiovisual English processing in native users. Brain, v.125, n.7, p. 15831593, 2002a.

MACSWEENEY, Mairéad et al. Neural correlates of British Sign Language comprehension: Spatial processing demands of topographic language. Journal of Cognitive Neuroscience, v.14, n.7, p. 1064-1075, 2002b.

MCCUllOUGH, Stephen; EMMOREY, Karen; SERENO, Martin. Neural organization for recognition of grammatical and emotional facial expressions in deaf ASL signers and hearing nonsigners. Brain Research, v.22, n.2, p.193-203, 2005. 
MEYER, Martin et al. fMRI reveals brain regions mediating slow prosodic modulations in spoken sentences. Human Brain Mapping, v.17, n.2, p.73-88, 2002. MITCHELL, Rachel L. et al. The neural response to emotional prosody, as revealed by functional magnetic resonance imaging. Neuropsychologia, v.41, n.10, p.1410-1421, 2003.

MOURA, Maria Cecília; LODI, Ana Claudia Balieiro; HARISSON, Kathryn Marie P. História e educação do surdo, a oralidade e o uso de sinais. IN: FILHO, O.L. Tratado de Fonoaudiologia. 2a ed. Ribeirão Preto: Tecmedd, 2005.

NARUMOTO, Jin et al. Attention to emotion modulates fMRI activity in human right superior temporal sulcus. Cognitive Brain Research, v.12, n. 2, p. 225-231, 2001.

NEVILLE, Helen J. et al. Cerebral organization for language in deaf and hearing subjects: biological constraints and effects of experience. Proceedings of the National Academy of Sciences, v.95, n.3, p. 922-029, 1998.

NEWMAN, Aaron J. et al. Prosodic and narrative processing in American Sign Language: an fMRI study. Neuroimage, v.52, n.2, p. 669-676, 2010.

OGAWA, Seiji et al. Brain magnetic resonance imaging with contrast dependent on blood oxygenation. Proceedings of the National Academy of Sciences, v.87, n. 24, p. 9868-72, 1990a.

OGAWA, Seiji et al. Oxygenation-sensitive contrast in magnetic resonance image of rodent brain at high magnetic fields. Magnetic Resonance in Medicine, v.14, n.1, p. 68-78, 1990 b.

POIZNER, H.; KLIMA, E.S.; BELLUGI, U. What the hands reveal about the brain. Cambridge, M.A.: The MIT Press, 1987.

QUADROS, Ronice Müller de; KARNOPP, Lodenir Becker. Lingua de sinais brasileira: estudos linguísticos. Porto Alegre: Arqmed, 2004.

ROSSET, Sara Regina Escorsi. Mapeamento de áreas de linguagem por meio da ressonância magnética funcional em indivíduos assintomáticos, pacientes com epilepsia e candidatos a neurocirurgia. Ribeirão Preto: USP, 2008. Tese (Doutorado) - Faculdade de Medicina da Universidade de São Paulo, Ribeirão Preto, 2008.

SAUSSURE, Ferdinand de. Curso de linguística geral. 20a ed. São Paulo: Cultrix, 1995. 
SÖDERFELDT, Björn et al. Signed and spoken language perception studied by positron emission tomography. Neurology, v.491, n.1, p.82-87, 1997.

SÖDERFELDT, Björn; RÖNNBERG, Jerker; RISBERG, Jan. Regional cerebral blood flow in sign language users. Brain and Language, v.46, n.1, p.59-68, 1994. SOUZA, Regina Maria de. Que palavra que te falta? Linguística e educação: considerações epistemológicas a partir da surdez. São Paulo: Martins Fontes, 1998. STOKOE, W. Sign language structure: an outline of the visual communication systems of the American deaf. 2 nd ed. Maryland, Linstok Press, 1960/1978. STURZBECHER, Marcio Junior. Detecção e caracterização da resposta hemodinâmica pelo desenvolvimento de novos métodos de processamento de imagens funcionais por ressonância magnética. Ribeirão Preto: USP, 2006. Dissertação (Mestrado) - Faculdade de Filosofia, Ciências e Letras da Universidade de São Paulo, Ribeirão Preto, 2006.

TALAIRACH, Jean; TOURNOUX, Pierre. Co-plannar stereotaxic atlas of the buman brain. New York: Thieme Medical Publishers, 1988.

${ }^{1}$ Psicóloga e coordenadora da Neuropsicologia Infantil do Centro de Cirurgia de Epilepsia de Ribeirão Preto (CIREP), no Hospital das Clínicas da Faculdade de Medicina (FMRPUSP). Possui Doutorado em Neurologia/Neurociências. Especialização em Reabilitação Cognitiva e Avaliação Neuropsicológica, pela Universidade de São Paulo.

${ }^{2}$ Trata-se do paradigma utilizado na maioria dos centros que realizam estudos com IRMf e consiste em períodos de atividade alternados por períodos iguais de repouso. Assim, ocorre a geração de estados "on" e "off da atividade neuronal, os quais se correlacionarão diretamente à intensidade perfusional (e, consequentemente, ao sinal BOLD) na área de interesse. Obtém-se, assim, um estado da atividade neuronal que estará, a princípio, correlacionada a um aumento de perfusão local. Esse método visa a localizar as regiões que responderam seletivamente à alternância entre a execução da atividade e os períodos de repouso. Sua maior vantagem está na capacidade de localização das áreas anatomofuncionais de interesse (DE ARAUJO, 2002; ROSSET, 2008).

${ }^{3} \mathrm{O}$ sistema de coordenadas Talairach é baseado no plano entre duas formações anatômicas típicas: a comissura anterior e a comissura posterior. Após a definição do plano de coordenadas, são geradas quatro linhas de referências que formam o sistema de grade Talairach, o qual é estabelecido com base nas máximas dimensões do cérebro. O passo 
seguinte, de demarcação, é utilizado para definir a periferia cortical. Depois da demarcação das dimensões máximas de cada cérebro, são realizadas contrações e expansões na imagem do cérebro, a fim de que ele preencha este sistema de grade. Por meio desses processos, é possível normalizar cada cérebro para uma estrutura padrão. 
